\title{
Vitamin D promotes the cisplatin sensitivity of oral squamous cell carcinoma by inhibiting LCN2-modulated NF-KB pathway activation through RPS3
}

\author{
Zixian Huang ${ }^{1,2}$, Yin Zhang ${ }^{1,3}$, Haigang Li ${ }^{4}$, Yufeng Zhou ${ }^{5}$, Qianyu Zhang ${ }^{1,2}$, Rui Chen ${ }^{1,2}$, Tingting Jin ${ }^{1,2}$, Kaishun Hu ${ }^{1,3}$, \\ Shihao Li ${ }^{2}$, Yan Wang ${ }^{2}$, Weiliang Chen ${ }^{2}$ and Zhiquan Huang ${ }^{1,2}$
}

\begin{abstract}
Chemoresistance is a major cause of cancer progression and the mortality of cancer patients. Developing a safe strategy for enhancing chemosensitivity is a challenge for biomedical science. Recent studies have suggested that vitamin D supplementation may decrease the risk of many cancers. However, the role of vitamin D in chemotherapy remains unknown. We found that vitamin D sensitised oral cancer cells to cisplatin and partially reversed cisplatin resistance. Using RNA-seq, we discovered that lipocalin 2 (LCN2) is an important mediator. Cisplatin enhanced the expression of LCN2 by decreasing methylation at the promoter, whereas vitamin D enhanced methylation and thereby inhibited the expression of LCN2. Overexpression of LCN2 increased cell survival and cisplatin resistance both in vitro and in vivo. High LCN2 expression was positively associated with differentiation, lymph node metastasis, and T staging and predicted a poor prognosis in oral squamous cell carcinoma (OSCC) patients. LCN2 was also associated with post-chemotherapy recurrence. Moreover, we found that LCN2 promoted the activation of NF-KB by binding to ribosomal protein S3 (RPS3) and enhanced the interaction between RPS3 and p65. Our study reveals that vitamin D can enhance cisplatin chemotherapy and suggests that vitamin D should be supplied during chemotherapy; however, more follow-up clinical studies are needed.
\end{abstract}

\section{Introduction}

Oral cancer is one of the most lethal malignant tumours in the world, and its incidence is increasing yearly ${ }^{1,2}$. It occurs in sites highly vascularises with blood and lymphatic vessels, which may lead to in situ recurrence and distant metastasis. Hence, the prognosis of oral cancer patients is poor.

\footnotetext{
Correspondence: Weiliang Chen (drchen@vip.163.com) or

Zhiquan Huang (hzhquan@mail.sysu.edu.cn)

'Guangdong Provincial Key Laboratory of Malignant Tumor Epigenetics and

Gene Regulation, Sun Yat-Sen Memorial Hospital, Sun Yat-Sen University,

Guangzhou, China

${ }^{2}$ Department of Oral and Maxillofacial Surgery, Sun Yat-sen Memorial Hospital, Sun Yat-sen University, Guangzhou, China

Full list of author information is available at the end of the article.

These authors contributed equally: Zixian Huang, Yin Zhang

Edited by A. Stephanou
}

The major type of oral cancer is oral squamous cell carcinoma (OSCC). Cisplatin is the first-line drug for postoperative chemotherapy in OSCC patients; however, $>30 \%$ of patients are initially insensitive to platinum-based chemotherapy, and the rest gradually become insensitive to previously effective drugs after several rounds of chemotherapy $y^{3,4}$. Intrinsic and acquired chemoresistance are major causes of the progression and mortality of oral cancer patients. Some strategies, such as combination chemotherapy and radiotherapy and the combination of different types of chemotherapeutic drugs, have been applied in patients. However, due to some common underlying mechanisms, chemo-resistant patients are usually insensitive to other therapy strategies ${ }^{5}$. Moreover, these combination therapy strategies could significantly

\section{(c) The Author(s) 2019}

(c) (i) Open Access This article is licensed under a Creative Commons Attribution 4.0 International License, which permits use, sharing, adaptation, distribution and reproduction cc. in any medium or format, as long as you give appropriate credit to the original author(s) and the source, provide a link to the Creative Commons license, and indicate if changes were made. The images or other third party material in this article are included in the article's Creative Commons license, unless indicated otherwise in a credit line to the material. If material is not included in the article's Creative Commons license and your intended use is not permitted by statutory regulation or exceeds the permitted use, you will need to obtain permission directly from the copyright holder. To view a copy of this license, visit http://creativecommons.org/licenses/by/4.0/. 
increase the side effects of chemotherapy, such as bone marrow suppression and the impairment of immune cells in the tumour microenvironment ${ }^{6,7}$. Therefore, how to effectively overcome chemotherapy resistance while minimising adverse effects is a continuing challenge for biomedical science.

Vitamin D is a kind of fat-soluble secosteroid (also known as 1,25-dihydroxy-vitamin D3 (1,25(OH)2D3)). It mediates numerous physiological functions, such as regulating calcium and phosphorus metabolism, maintaining stable plasma calcium and phosphorus levels and participating in the growth of teeth and bones ${ }^{8}$. Clinically, it is often used for the treatment of rickets and osteoporosis.

In recent years, evidence from preclinical and clinical studies has suggested that vitamin D supplementation may decrease the risk of many cancers ${ }^{9}$.

Vitamin D has been found to have antitumour effects, suggesting that it might inhibit the progression of rectal cancer $^{10}$, breast cancer ${ }^{11}$, pancreatic cancer ${ }^{12,13}$, head and neck cancers ${ }^{14}$ and other cancers. Urashima ${ }^{15}$ suggested that the higher the serum level of vitamin $\mathrm{D}$ is, the better is the prognosis of rectal cancer patients. However, the effects of vitamin D in chemotherapy remain unknown.

Here, we report that vitamin D could promote cisplatin sensitivity in OSCC cells and xenografted tumours. By analysing RNA-seq data in OSCC cells treated with vitamin D and cisplatin, we found that lipocalin 2 (LCN2) was an important modulator of cisplatin sensitivity regulated by vitamin D. Moreover, we discovered that cisplatin could induce demethylation of the LCN2 promoter and thus increase its expression, and vitamin D could reverse this process. Both loss-of-function and gain-of-function experiments revealed that LCN2 could decrease the sensitivity of cisplatin in OSCC cells and xenografted tumours. Mechanistically, LCN2 regulates nuclear factor kappa B (NF-kB) activation by binding to RPS3 and enhancing the interaction between RPS3 and P65. Furthermore, we demonstrated that LCN2 expression in OSCC patients could affect postoperative survival time and is associated with recurrence after chemotherapy.

\section{Results}

\section{Vitamin D promoted cisplatin-based chemosensitivity in oscC}

To investigate the effects of vitamin D on cisplatinbased chemosensitivity, OSCC cells (CAL-27 \& SCC-9) were pretreated with $30 \mathrm{nM}$ vitamin $\mathrm{D}$ for 3 days and then treated with dose-gradient cisplatin ${ }^{16}$. MTS assay showed that vitamin $\mathrm{D}$ pretreatment resulted in the enhanced chemosensitivity of OSCC cells (Fig. 1a). Next, vitamin Dpretreated CAL-27 and SCC-9 cells were treated with $10 \mu \mathrm{M}$ and $15 \mu \mathrm{M}$ cisplatin, respectively. The cytotoxicity assay (lactate dehydrogenase (LDH) release, Fig. 1b) and flow cytometry (annexin V/PI, Fig. 1c) were used to detect cell death rates. Vitamin D had no significant effect on the OSCC cell death rate. However, in combination with cisplatin, it induced greater cell death than did cisplatin alone. Moreover, in CAL-27RE cells (the cisplatinresistant cell line of CAL-27), vitamin D partially reversed cisplatin resistance (Fig. 1sA).

Subsequently, CAL-27 cells were used to establish a nude mouse xenograft model. When the xenograft grew to $\sim 60 \mathrm{~mm}^{3}$, cisplatin and vitamin $\mathrm{D}$ were intraperitoneally injected, and the tumour volume was recorded (Fig. 1d). Although xenograft growth was slightly lower under vitamin D injection, the difference from control growth was not significant. Cisplatin inhibited the growth of tumours, and greater inhibition was observed when cisplatin was applied in combination with vitamin D. These data showed that vitamin D could enhance the sensitivity of OSCC to cisplatin and reduce cisplatin resistance.

\section{LCN2 was up-regulated after cisplatin treatment and down-regulated after vitamin $D$ treatment}

To explore the mechanism by which vitamin $\mathrm{D}$ suppresses cisplatin resistance in OSCC, we pretreated CAL27 cells with $5 \mu \mathrm{M}$ cisplatin and $30 \mathrm{nM}$ vitamin $\mathrm{D}$ for $36 \mathrm{~h}$ (Fig. 2sG). Then, total RNA was extracted for highthroughput sequencing. GO analysis showed the genes differentially expressed (DE) following vitamin D and cisplatin treatment shared some common biological process terms, such as response to lipopolysaccharide and response to drug (Fig. 2b, c). Many genes showed differential expression following treatment with vitamin $\mathrm{D}$ and cisplatin, in accordance with the phenotype in OSCC cells. Lipocalin-2 (LCN2) was one of the most significantly changed genes after both vitamin D and cisplatin treatment. When both vitamin $\mathrm{D}$ and cisplatin were administered, LCN2 expression differed little from that under control treatment, which revealed vitamin D could abrogate the up-regulation induced by cisplatin (Fig. 2a). LCN2, also known as oncogene $24 \mathrm{p} 3$ or neutrophil gelatinase-associated lipocalin (NGAL), belongs to the lipocalin family. Recently, LCN2 was reported to potentially play important roles in tumour progression and chemoresistance in many kinds of cancer ${ }^{17}$. Hence, we selected LCN2 for further analysis.

We verified the RNA-seq results by RT-qPCR and western blot, and similar results were obtained for both CAL-27 and SCC-9 cells (Figs. 2d, e and 1sF). We further analysed the expression of LCN2 in cisplatin-resistant cells. Compared with their expression in CAL-27 cells, LCN2 mRNA and protein expression was up-regulated in CAL-27RE cells (Fig. 1sB, C). We performed LCN2 immunohistochemical staining on the aforementioned OSCC xenografts (Fig. $2 \mathrm{f}$ and Table 1). The highexpression rate of LCN2 in tumour specimens after 


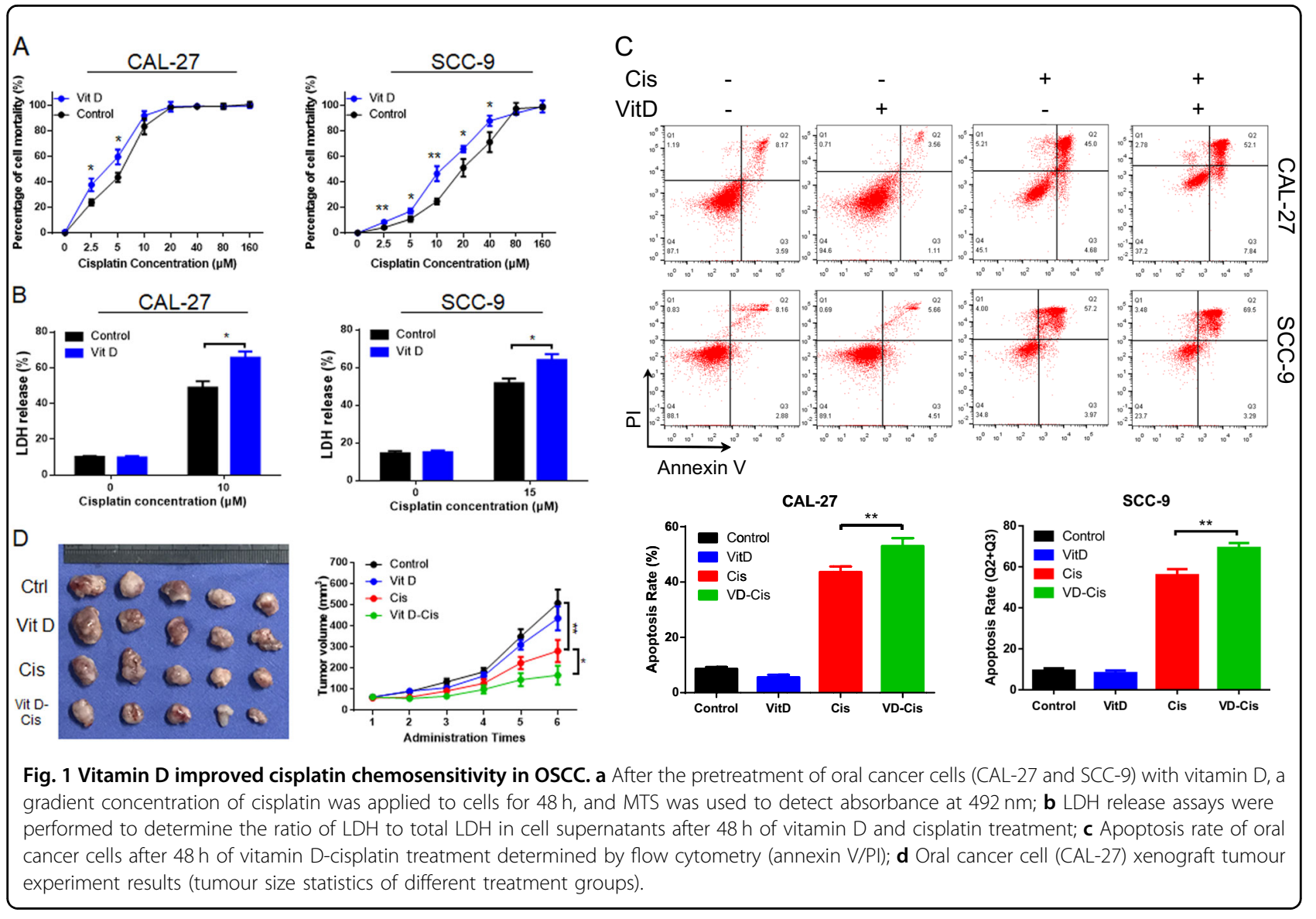

cisplatin treatment was $100 \%(5 / 5)$, while the control group and the vitamin D treatment group showed weak or no expression. When cisplatin was combined with vitamin $D$, the expression of LCN2 in the xenografts was partially inhibited (3/5). The trend in vivo was similar to that in vitro.

Vitamin D receptor (VDR) is a ligand-inducible transcription factors. To investigate whether LCN2 is a target gene of VDR, we knocked down VDR by siRNAs. QPCR and western blot analysis revealed no pronounced changes after the successfully silencing of VDR (Fig. 2sD-F). These results imply that vitamin $\mathrm{D}$ regulates LCN2 expression via a VDR-independent mechanism.

Chemotherapy sensitivity may be due to changes in gene expression caused by epigenetic changes such as DNA methylation at the promoter after treatment ${ }^{18}$. Methylation at promoters has been reported to play an important role in regulating LCN2 expression ${ }^{17}$. So we evaluated methylation status after vitamin $\mathrm{D}$ and cisplatin treatment. Cisplatin treatment decreased the degree of methylation, whereas treatment with vitamin $\mathrm{D}$ increased the methylation of the LCN2 promoter. Moreover, vitamin $\mathrm{D}$ reversed the aberrant methylation caused by cisplatin, which ultimately reduced the expression of LCN2 (Fig. 2g). These results indicate that vitamin $\mathrm{D}$ and cisplatin regulate the expression of LCN2 by regulating LCN2 promoter methylation.

\section{LCN2 expression is associated with cisplatin insensitivity in OSCC cells}

To investigate the relationship between the expression level of LCN2 and the effect of cisplatin on OSCC, we constructed LCN2-overexpressing cell lines (LCN2-ov) and knockdown cell lines (sh-LCN2) of CAL-27 and SCC9 cells, which were verified by PCR (Fig. 3sA) and western blot analysis (Figs. 3a and 3sB). When LCN2 expression was down-regulated, OSCC cell cisplatin sensitivity was up-regulated (Fig. 3b). At a given concentration, cisplatin induced a high rate of death in sh-LCN2 cells (Figs. 3d and $3 \mathrm{sC}$ ); when LCN2 was overexpressed, OSCC cells were significantly more resistant to cisplatin (Fig. 3c). Flow cytometry (Figs. 3e and 3sD) showed that the mortality of LCN2-overexpressing cells was decreased relative to that of shLCN2 cells at the same concentration of cisplatin. The down-regulation of LCN2 also inhibited the chemoresistance of CAL-27RE cells to cisplatin (Fig. 1sD). This finding indicated that LCN2 was an important regulator of cisplatin sensitivity in OSCC cells.

Next, two additional xenograft models were established using the CAL-27 LCN2 stable cell line (shLCN2 \& 


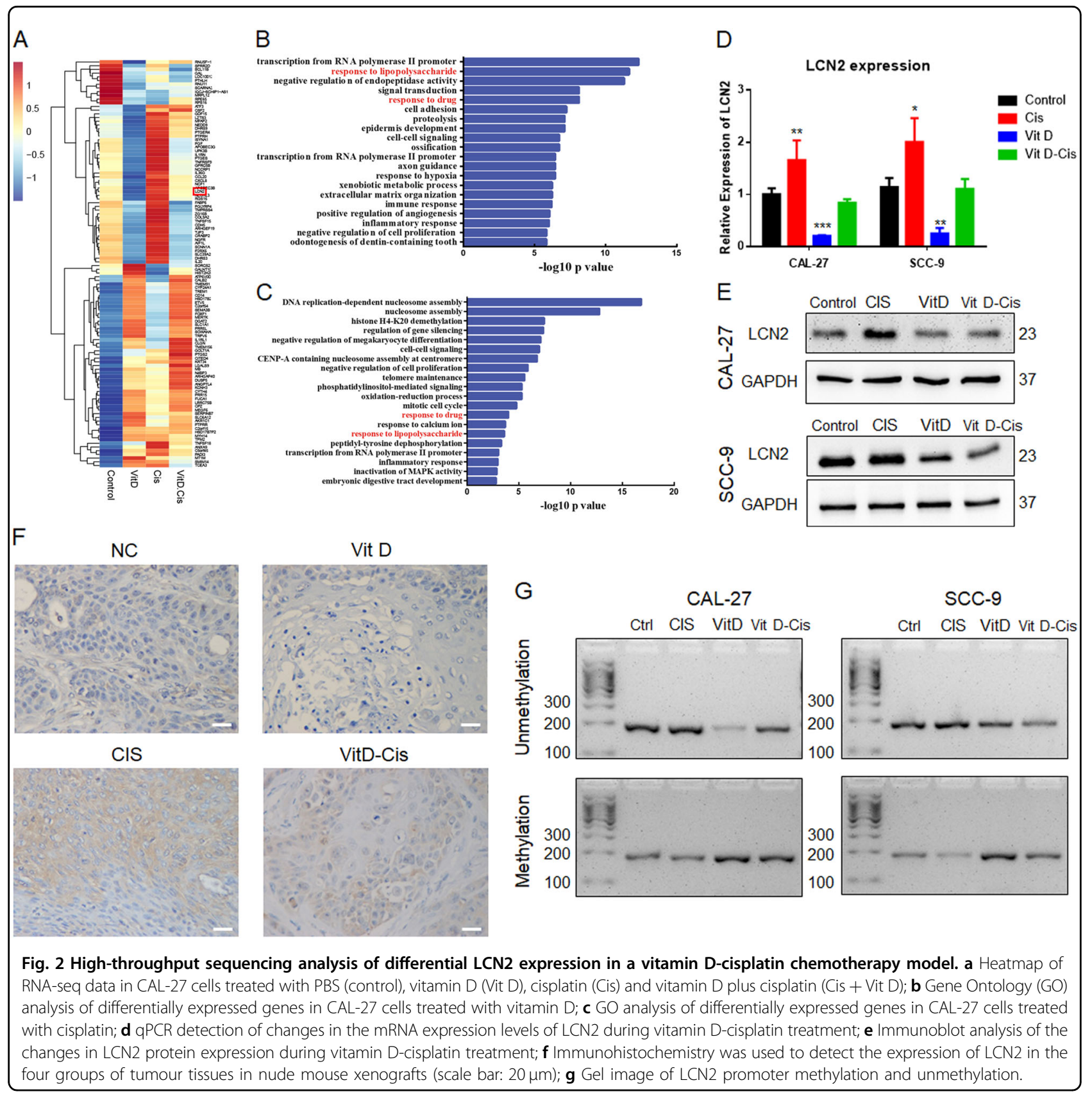

LCN2-ov), and cisplatin chemotherapy was administered. The results showed that upon silencing LCN2 expression, tumour growth was inhibited (Figs. 3f and 3sE), which was in accordance with the in vitro experiments. After cisplatin treatment, both the control group and the shLCN2 group showed decreasing trends, but the latter group showed a greater decrease, indicating a higher cisplatin sensitivity of sh-LCN2 cells. The opposite trend was observed in LCN2-overexpressing xenografts. In the LCN2-ov group (Figs. 3g and 3sF) cisplatin chemotherapy had little effect on tumour size, whereas cisplatin significantly reduced the size of tumours in the control group.

These data reveal that LCN2 is an important regulatory target for tumour chemosensitivity during cisplatin therapy in OSCC and that the inhibition of LCN2 can significantly promote chemotherapy effects.

\section{Vitamin D suppressed NF-кB phosphorylation via LCN2 inhibition}

The NF- $\kappa B$ pathway is a fundamental pathway in cancer. The constitutive activation of NF- $k B$ has been 
Table 1 Association of LCN2 expression with the features of Xenograft.

\begin{tabular}{llll}
\hline Group & \multicolumn{2}{l}{ LCN2 expression } & P value \\
\cline { 2 - 3 } & High & Low & \\
\hline Without Vit D & & & \\
Control & 1 & 4 & 0.048 \\
Cisplatin & 5 & 0 & \\
Vit D applied & & & \\
Control & 0 & 5 & 0.167 \\
Cisplatin & 3 & 2 & \\
\hline
\end{tabular}

demonstrated in various types of cancer. NF- $\mathrm{kB}$ can result in chemoresistance by activating many anti-apoptotic genes, such as FLIP, c-IAP1/2 and XIAP ${ }^{19}$. Our previous worked indicated that vitamin $\mathrm{D}$ could inhibit the NF- $\mathrm{KB}$ pathway ${ }^{16,20}$. Moreover, KEGG analysis of RNA-seq data from CAL-27 cells showed that cisplatin-induced genes were enriched in the NF- $\kappa$ B pathway (Fig. 4sD).

So we explored whether NF- $k B$ play important roles in LCN2-mediated cisplatin resistance. We found that cisplatin stimulated the activation of NF- $\mathrm{KB}$ (enhanced P65S536 phosphorylation), and vitamin $\mathrm{D}$ inhibited the phosphorylation of NF- $\mathrm{kB}$; thus, the activation of p-p65 caused by cisplatin stimulation decreased in the VitD-cis group (Figs. 4a and $4 \mathrm{sH}$ ). The vitamin $\mathrm{D}-\mathrm{NF}-\mathrm{kB}$ regulatory trend is consistent with $\mathrm{LCN} 2$, as shown in

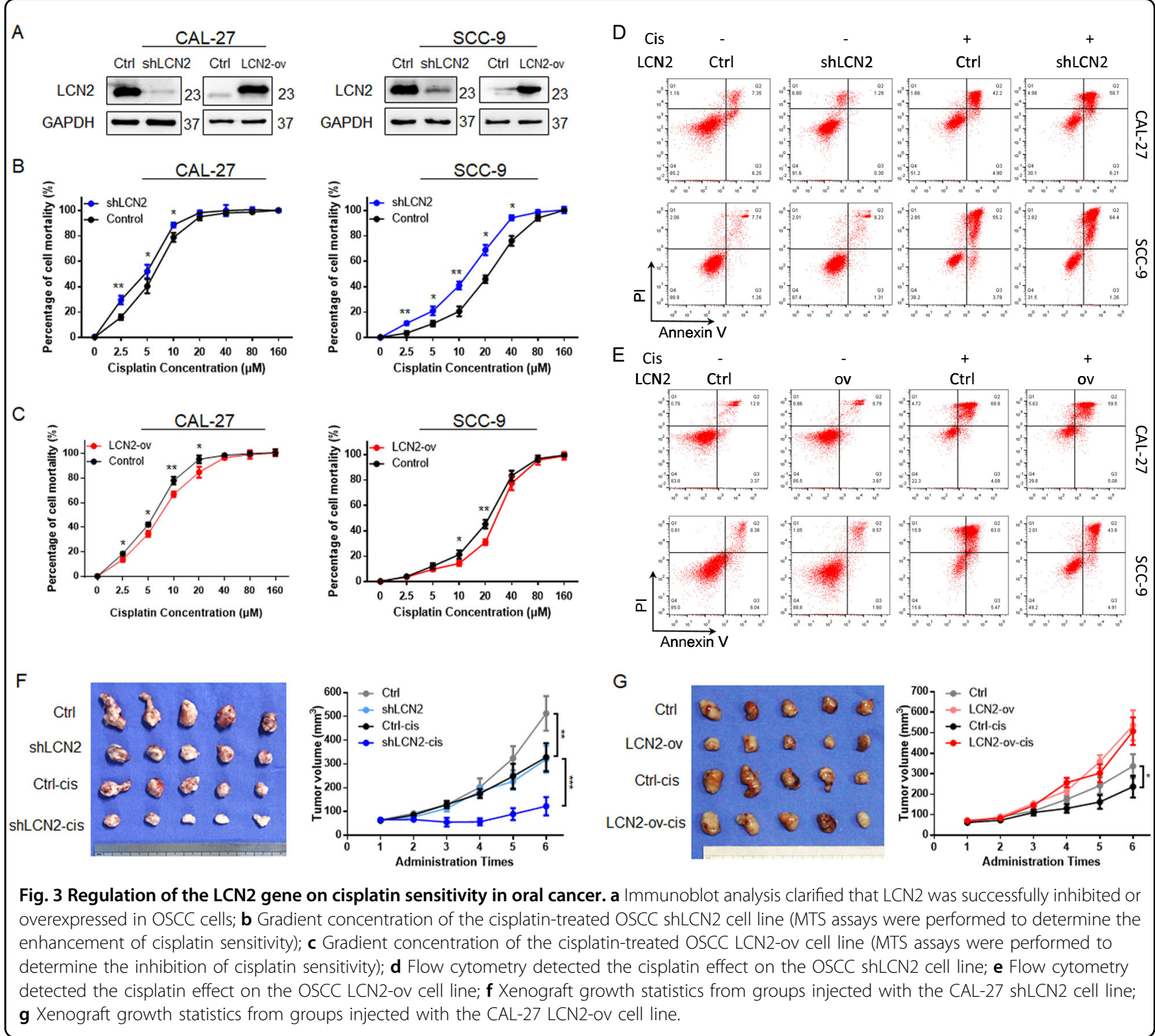


A

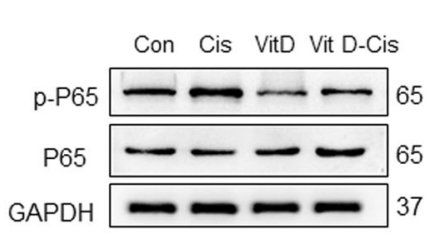

D

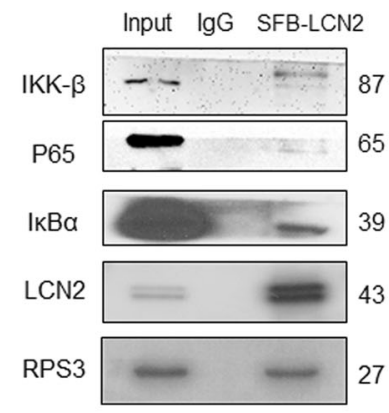

B

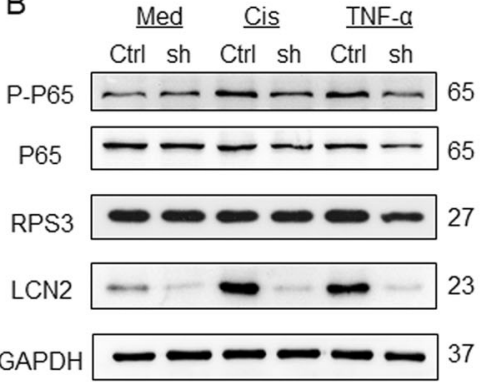

E Input IgG RPS3-IP

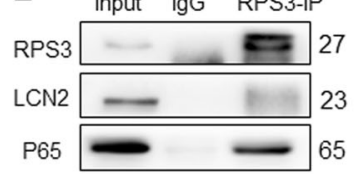

$\mathrm{F}$

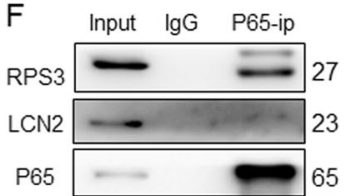

C

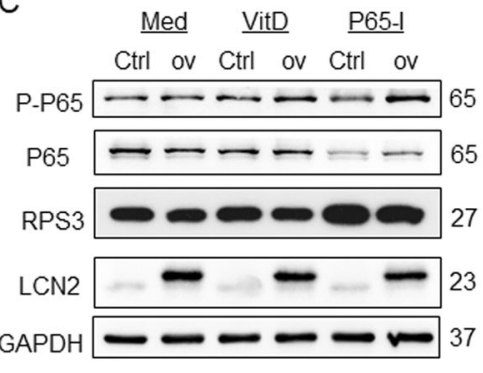

G

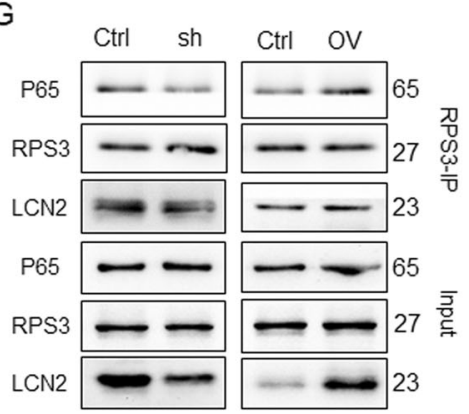

Fig. 4 The LCN2-RPS3 complex inhibited P65 phosphorylation. a Immunoblot analysis of the effects of vitamin D and cisplatin treatment on NFKB (P65) activation ( $p$-p65); $\mathbf{b}$ After the inhibition of LCN2 expression, cisplatin and TNF-a stimulation had a weak effect on the expression of P-P65, whereas the expression of P-P65 in the control group was significantly increased. The treatment had little effect on the expression of RPS3; c After the upregulation of LCN2, vitamin D and NF-KB inhibitors (BAY-11-7085, P65-I in this legend) exhibited a decreased ability to inhibit P-P65 expression, and this treatment had little effect on RPS3 expression; d After the SFB tag was fused to LCN2, IP experiments were performed using an SFB tag, which revealed weak binding between LCN2 and the NF-KB pathway; e Endogenous RPS3-IP experiment: interaction between RPS3 and LCN2 and P65; f Endogenous NF-KB IP assay: NF-KB binds directly to RPS3 but not LCN2; $\mathbf{g}$ The RPS3-IP experiment was performed in LCN2 stable cell lines: the binding between RPS3 and P65 was decreased after LCN2 inhibition; the binding ability between RPS3 and P65 was enhanced after LCN2 expression was up-regulated. Med, culture medium; Cis, cisplatin; P65-I, p65 inhibitor.

Fig. 2f. Therefore, we further speculate that LCN2 is an important regulator between vitamin $\mathrm{D}$ and NF- $\mathrm{kB}$.

When LCN2 was inhibited, cisplatin or TNF- $\alpha$ stimulation slightly increased the phosphorylation level of p65, whereas the expression of p-p65 was enhanced in the control group (Figs. 4b and 4sI). Similarly, when LCN2 was overexpressed, p-P65 inhibition by vitamin D and the NF-kB inhibitor (P65-I, BAY-11-7085) was attenuated (Figs. 4c and 4sJ). Hence, LCN2 is a key protein in the regulation of NF- $\mathrm{KB}$ by vitamin $\mathrm{D}$ and cisplatin.

\section{The LCN2-RPS3 complex regulated P65 activation}

To investigate the mechanism by which LCN2 regulates NF- $\mathrm{kB}$, we first used an endogenous antibody for immunoprecipitation (IP) experiments. However, we did not detect an association between LCN2 and the NF- $\mathrm{KB}$ pathway (Fig. 4sA). Subsequently, we constructed an SFB tag in the LCN2 N-terminus and used it for IP experiments $^{21}$, which revealed that LCN2 has a weak linkage with NF-KB signalling pathway protein (Fig. 4d). Therefore, we hypothesised that regulatory proteins function in interactions between LCN2 and the NF- $\mathrm{kB}$ pathway.
Next, we used mass spectrometry to identify the proteins involved in the interaction between LCN2 and NF$\kappa B$. Discovered by mass spectrometry (Fig. 4sE-G), an important NF-kB regulator, ribosomal protein S3 (RPS3) $^{22}$, was found to bind to LCN2.

We verified the mass spectrometry result by IP and detected strong interactions of LCN2 and RPS3 through both SFB-tagged and endogenous antibody of LCN2 (Figs. $4 \mathrm{~d}$ and 4SA). A direct interaction between RPS3 and the NF- $\kappa B$ pathway was detected (Figs. 4e, f, and $4 \mathrm{SB}$ ), which has been reported previously ${ }^{23}$. Previous studies showed NF- $\kappa B$ activation is affected by the binding of RPS3 to $\mathrm{P}^{24}{ }^{24}$, so we speculated that LCN2 may interact with P65 indirectly: LNC2 may bind directly to RPS3 and promote the interaction between RPS3 and P65, thereby regulating NF- $\kappa B$ activation.

To test this hypothesis, we conducted coimmunoprecipitation (Co-IP) assays in LNC2overexpression and knockdown stable cell lines (Fig. 4c). We found that upon silencing LCN2, the interaction between RPS3 and P65 was reduced; when LCN2 was overexpressed, RPS3 and P65 binding increased. Moreover, vitamin $\mathrm{D}$ and cisplatin treatment yielded no 
Table 2 Association of LCN2 expression with the features of OSCC patients.

\begin{tabular}{|c|c|c|c|}
\hline \multirow[t]{2}{*}{ Characteristics } & \multicolumn{2}{|c|}{ LCN2 expression } & \multirow[t]{2}{*}{$P$ value } \\
\hline & High (\%) & Low (\%) & \\
\hline \multicolumn{4}{|l|}{ Age } \\
\hline$\leq 40$ & $11(42.31)$ & $15(57.69)$ & \\
\hline$>40$ & $13(33.33)$ & $26(66.67)$ & 0.4705 \\
\hline \multicolumn{4}{|l|}{ Gender } \\
\hline Male & $14(36.84)$ & $24(63.16)$ & \\
\hline Female & $10(37.04)$ & $17(62.96)$ & 0.9874 \\
\hline \multicolumn{4}{|l|}{ Differentiation } \\
\hline Well & $6(22.22)$ & $21(77.78)$ & \\
\hline Moderate & $8(36.36)$ & $14(63.64)$ & \\
\hline Poor & $10(62.50)$ & $6(37.50)$ & 0.0087 \\
\hline \multicolumn{4}{|c|}{ Lymphatic metastasis } \\
\hline No & $7(21.88)$ & $25(78.12)$ & \\
\hline Yes & $17(51.52)$ & $16(48.48)$ & 0.0128 \\
\hline \multicolumn{4}{|l|}{ Clinical stage (T) } \\
\hline 1 & $2(11.76)$ & $15(88.24)$ & \\
\hline 2 & $9(40.91)$ & $13(59.09)$ & \\
\hline 3 & $8(50.00)$ & $8(50.00)$ & \\
\hline 4 & $5(50.00)$ & $5(50.00)$ & 0.0233 \\
\hline
\end{tabular}

Lcn2 positive 24 (36.92\%), negative 41 (63.08\%)

significant changes in RPS3 expression (Fig. 4sC). These data suggest that LCN2 could activate the NF- $\mathrm{kB}$ pathway by enhancing the interaction between RPS3 and P65.

The expression of LCN2 is associated with the prognosis of OSCC patients

We performed LCN2 immunohistochemical staining analysis on specimens from patients with OSCC who were not previously treated with radiotherapy or chemotherapy (Table 2 and Fig. 5a-f). The high-expression rate of LCN2 was low $(36.9 \%, 24 / 65)$ in this group, and LCN2 expression did not correlate with patient age or gender but was positively correlated with lymph metastasis, clinical stage (T stage) and malignancy (differentiation). Patients with OSCC who had high LCN2 expression experienced a shorter survival time (Fig. $5 \mathrm{~g}$ ). Hence, LCN2 is one of the potential indicators of a poor prognosis in clinical patients.

Is LCN2 expression related to recurrence after chemotherapy? To address this question, we collected samples from 36 patients with recurrent OSCC and performed immunohistochemical staining. The highexpression rate of LCN2 in recurrent patients was $69.4 \%$
(25/36). Among them, 10 patients with recurrence could track their primary tumour samples: the initial rate of LCN2 was $60.0 \%(6 / 10)$, and the recurrence rate of LCN2 was as high as $80.0 \%(8 / 10)$. Low-expression LCN2 patients had elevated expression after chemotherapy; two patients with low LCN2 expression did not receive chemotherapy; two patients had high LCN2 expression and did not experience recurrence after chemotherapy; and four patients had high LCN2 expression and relapsed after chemotherapy.

Among the relapsed patients, 20 patients were treated with chemotherapy, and 17 patients were positive for LCN2 after recurrence $(85.0 \%, 17 / 20)$. The correlation analysis confirmed that in patients with relapse, high LCN2 expression was associated with chemotherapy (Table 3), and chemotherapy induction promoted high LCN2 expression. There was a difference in LCN2 expression between relapsed patients and primary patients (Table 4). However, in patients with recurrence, there was no significant correlation between the expression of LCN2 and survival time (Fig. 5h), which may be related to the small sample size. Further research needs to expand the sample size to more clearly define the relationship between LCN2 expression and survival in oral cancer patients with recurrence.

In conclusion, LCN2 was a key molecule responsible for drug sensitivity during cisplatin chemotherapy in OSCC. Vitamin D inhibited NF- $\mathrm{KB}$ activation by suppressing LCN2, which enhanced the cisplatin chemotherapeutic effects on OSCC (Fig. 6).

\section{Discussion}

The antitumour effect of vitamin D is a popular research topic. Epidemiological studies have compared the relationship between serum vitamin $D$ levels and prognosis in patients with breast cancer ${ }^{25}$ and rectal cancer $^{26}$ and have found that the higher the serum level of vitamin $\mathrm{D}$ is, the better is patient prognosis. The regulatory relationship between vitamin $\mathrm{D}$ and tumours has not yet been elucidated, and its mechanism has not been clarified. Vitamin D enters cells by two pathways: the steroid receptor pathway and the direct entry pathway. Vitamin D receptor (VDR) is a membrane receptor for vitamin D. However, whether VDR is a tumour suppressor or an oncogene remains unclear ${ }^{27-29}$. The VDR expressions in cisplatin and vitamin $\mathrm{D}$ treatments failed to show significant changes (Fig. 2sA-C). Hence, the disputed effects of VDR and vitamin D on cancer cells led us to postulate that the antitumour function of vitamin $\mathrm{D}$ is not solely borne by VDR and that other molecules are present.

In this study, we identified LCN2 as a potential regulator of vitamin D. LCN2, also known as neutrophil gelatinase-associated lipocalin (NGAL), is a tumour- 

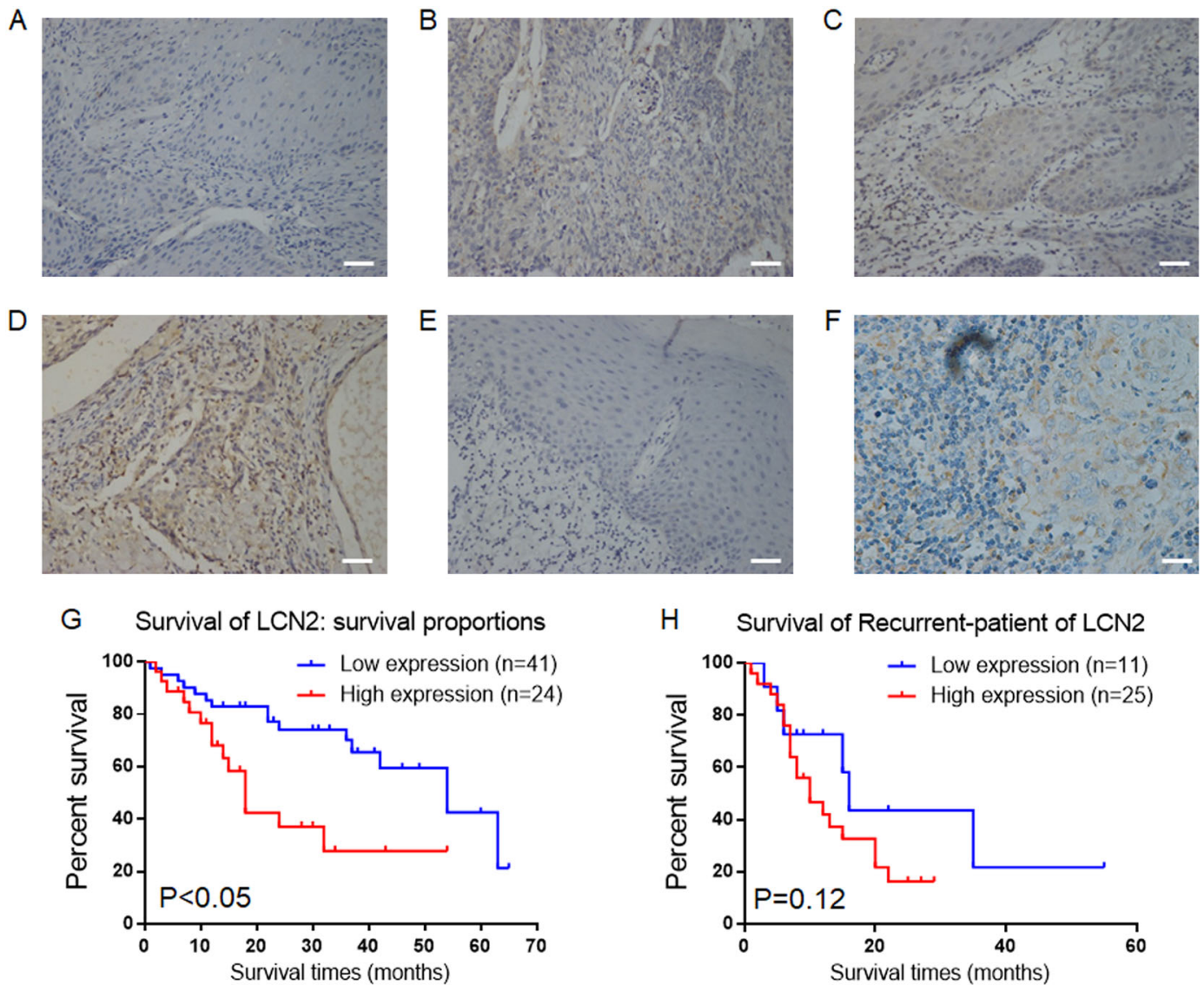

Fig. 5 The expression of LCN2 is associated with the poor prognosis of patients with OSCC. a Immunohistochemical analysis of LCN2 expression (grade 0) in well-differentiated OSCC; b Immunohistochemical analysis of LCN2 expression (grade 1) in well-differentiated OSCC; c Immunohistochemical analysis of LCN2 expression (grade 2) in moderately differentiated OSCC; $\mathbf{d}$ Immunohistochemical analysis of LCN2 expression (grade 3) in poorly differentiated OSCC; e Negative LCN2 expression in ANC tissue; f Positive LCN2 expression in lymphatic metastases; $\mathbf{g}$ Kaplan-Meier estimates of the overall survival of patients with LCN2 expression; $\mathbf{h}$ Kaplan-Meier estimates of the overall survival of patients with LCN2 expression who experienced recurrence (scale bar: $20 \mu \mathrm{m}$ ).

promoting gene ${ }^{30}$. LCN2 expression regulates the cisplatin sensitivity of OSCC. In the vitamin D-cisplatin chemotherapy model, cisplatin promoted LCN2 expression, whereas vitamin D inhibited LCN2 expression and partially reversed cisplatin-induced LCN2 upregulation. The tumour xenograft model showed that vitamin D inhibited the expression of LCN2 in the tumour and enhanced cisplatin sensitivity, while the tumour with high LCN2 expression was not sensitive to cisplatin. Clinically, LCN2 was positively associated with differentiation, lymph node metastasis, $\mathrm{T}$ staging and a poor prognosis in OSCC patients. Patients with postoperative recurrence, especially those who relapsed after chemotherapy, exhibited a statically significant upregulation of LCN2. This finding was validated with our laboratory findings, clarifying that LCN2 is a tumour chemosensitive gene and has potential as a predictor of tumour recurrence ${ }^{31}$. Furthermore, we experimentally proved that the regulation of LCN2 and VDR genes is independent of each other. VDR inhibition had no significant effect on VDR expression. In summary, we believe that the antitumour regulation of vitamin $\mathrm{D}$ is attributed to the inhibition of LCN2.

However, how does vitamin D suppress LCN2 expression? Chemotherapy sensitivity may be due to changes in gene expression caused by epigenetic changes, such as DNA methylation at the promoter during treatment ${ }^{32}$. Cisplatin kills cancer cells by binding to DNA and inducing DNA damage. Recent studies have revealed that mismatch repair proteins can recruit DNA methylation enzymes at the DNA damage site and change the methylation status ${ }^{33}$. A reversal of the aberrant methylation of key cisplatin resistance-related genes may provide a novel solution to chemotherapy resistance. In this study, vitamin D promoted LCN2 promoter methylation and reduced LCN2 expression. Furthermore, the unmethylation of LCN2 was associated with malignant tumour characterisation and a poor 


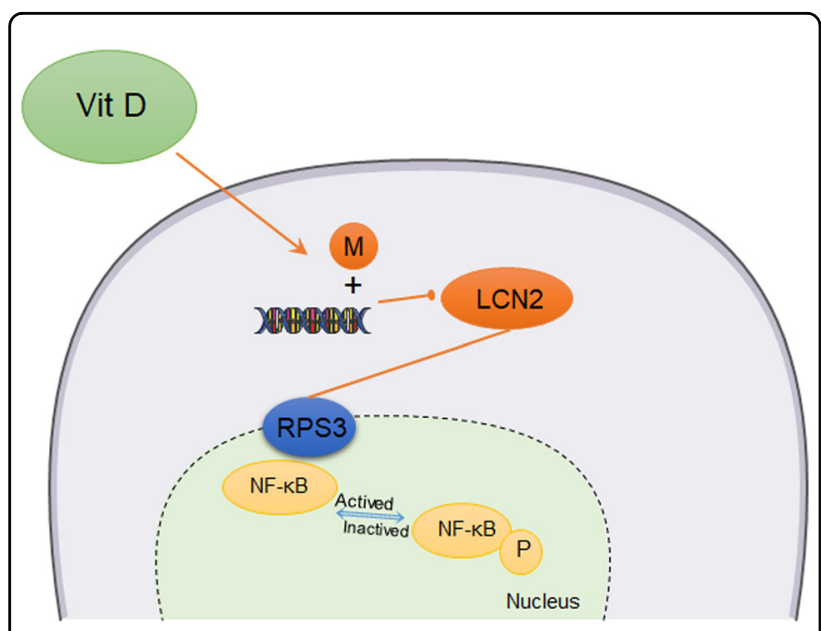

Fig. 6 Schematic model of how vitamin D promotes the cisplatin sensitivity of oral cancer by inhibiting the LCN2-RPS3-NF-KB pathway. In OSCC cells, vitamin D promoted LCN2 promoter methylation to inhibit LCN2 protein expression, which reduced LCN2RPS3 binding and thus reduced RPS3-NF-KB binding. The formation of the NF-KB complex ultimately reduced the activation of NF-KB, increasing the chemotherapeutic sensitivity of the tumour to cisplatin.

Table 3 Differential expression of LCN2 in patients with primary or recurrent OSCC.

\begin{tabular}{lllll}
\hline Characteristics & \multicolumn{2}{l}{ LCN2 expression } & $t$ & $P$-value \\
\cline { 2 - 3 } & High & Low & & \\
\hline OSCC patients & & & & \\
Primary & 24 & 41 & & \\
Recurrent & 25 & 11 & 3.263 & 0.002 \\
\hline
\end{tabular}

Table 4 Correlation between LCN2 and cisplatinchemotherapy in recurrent samples.

\begin{tabular}{lllll}
\hline Characteristics & \multicolumn{2}{l}{ LCN2 expression } & $R^{2}$ & $P$-value \\
\cline { 2 - 3 } & High Low & & \\
& & & \\
\hline
\end{tabular}

Chemotherapy

\begin{tabular}{lllll} 
Yes & 17 & 3 & & \\
No & 8 & 8 & 0.143 & 0.023 \\
\hline
\end{tabular}

prognosis, which may have resulted from the antitumour effect of vitamin D.

Vitamin D can regulate a variety of important pathways $^{34-37}$. Among these, the NF- $\mathrm{KB}$ pathway plays an important role in the regulation of cisplatin sensitivity. $\mathrm{NF}-\mathrm{kB}$ is present in almost all cells and has five family proteins $^{38,39}$ : RelA (p65), RelB, c-Rel, p50 and p52. The most common RelA (p65) C-terminus has a transactivation domain (TD) that activates the target gene. The sustained activation of NF-kB, represented by P65 phosphorylation, is an important mechanism for tumourigenesis, drug resistance regulation, and tumour self-defence ${ }^{40}$. The inhibition of NF- $\mathrm{KB}$ activation is beneficial to the treatment of tumours ${ }^{41}$. In this study, we found that once LCN2 was inhibited, the effect of NF- $\mathrm{KB}$ activation by cisplatin or TNF- $\alpha$ was weakened. When LCN2 was up-regulated, the inhibition of NF-kB by vitamin D or the NF- $\mathrm{KB}$ inhibitor was also attenuated.

These observations led to the question of whether there an intermediate regulatory factor between LCN2 and NF- $\mathrm{KB}$ or whether LCN2 acts directly on transcriptional activation ${ }^{42}$. When the IP assay was performed using an endogenous LCN2 antibody and SFBfusion-LCN2, weak binding of LCN2 to NF- $\mathrm{kB}$ was observed, suggesting a regulatory factor between LCN2 and NF- $\mathrm{kB}$. Mass spectrometry revealed that RPS3 may be the key to regulation. RPS3, which is located upstream of the NF- $\mathrm{kB}$ regulatory gene, activates the NF- $\kappa B$ pathway by interacting with the NF- $\kappa B$ com$\mathrm{plex}^{43}$. The interaction among RPS3, LCN2 and NF- $\mathrm{KB}$ is essential for subsequent regulation. When LCN2 expression was decreased, it affected the binding between RPS3 and NF- $\mathrm{kB}$, thereby reducing the activation of NF-kB.

A new vitamin D-LCN2-RPS3-NF- $\mathrm{kB}$ regulatory model was established. Vitamin D promoted LCN2 promoter methylation to inhibit LCN2 protein expression, reduced LCN2-RPS3 binding, and thus reduced RPS3-NF- $k B$ binding. The formation of the NF- $k B$ complex ultimately reduced the activation of NF- $\mathrm{kB}$, thereby increasing the chemotherapeutic sensitivity of the tumour to cisplatin.

In conclusion, the present study identified a new vitamin $\mathrm{D}$ subregulator, $\mathrm{LCN} 2$, that could enhance the cisplatin effect on OSCC by inhibiting NF- $\mathrm{KB}$ activation. The downregulation of LCN2 was capable of promoting OSCC chemosensitivity in vitro and in vivo. More importantly, high LCN2 expression predicted a poor prognosis in OSCC patients and was associated with OSCC chemosensitivity and postoperative recurrence. Therefore, LCN2 could be used as a potential target for tumour chemosensitivity. At the same time, based on this study, the use of vitamin D is important for the inhibition of LCN2 and other genes, which benefited the sensitisation of tumour cells to chemotherapy. Although the initial expression of the LCN2 protein was low, the subsequent increase caused by chemotherapy cannot be ignored. Once LCN2 was highly expressed, the vitamin $\mathrm{D}$ inhibitory effect on LCN2-NF- $\mathrm{B}$ was weakened, and the chemosensitivity of tumour cells was reduced. Therefore, vitamin D should be taken after the start of 
chemotherapy, but more follow-up clinical studies are needed to support this hypothesis.

\section{Materials and methods Antibodies}

Mouse monoclonal anti-FLAG (\#F3165) was purchased from Sigma. Antibodies against p-P65 (\#3033), P-65 (\#8242), RPS3 (\#9538), IKK- $\beta$ (\#8943), ІкB $\alpha$ (\#4814) and GAPDH (\#D16H11) were purchased from Cell Signaling Technology. VDR (\#MABS2028) and LCN2 (\#AB2267) antibodies were purchased from Millipore. Secondary antibodies, including goat anti-mouse IgG-HRP (sc-2005) and goat anti-rabbit IgG-HRP (sc-2004), were purchased from Santa Cruz Biotechnology.

\section{Reagents}

The vitamin D metabolite 1,25D3 (Sigma, USA) was dissolved at a concentration of $400 \mu \mathrm{M}$ in anhydrous alcohol (AA) for preservation. Immediately prior to use, the stock was diluted to a final concentration of $30 \mathrm{nM}$ in culture medium. An NF- $\mathrm{B}$ B inhibitor (BAY 11-7082) was purchased from Beyotime (Shanghai, China). Other chemicals were purchased from Sigma. All of the culture media (DMEM for CAL-27/27RE, DMEM-F12 for SCC-9) and foetal bovine serum (FBS) were purchased from Bioind.

\section{Plasmids}

Human LCN2 and LCN2 short hairpin RNAs (shRNAs) were purchased from Vigene Biosciences, and the sequence of the shRNA was as follows:

GAGCTGACTTCGGAACTAATTCAAGAGATTAGT TCCGAAGTCAGCTCTTTTTT. pMD2.G (\#12259, Addgene) and psPAX2 (\#12260, Addgene) were used as packaging vectors.

To generate overexpression cell lines, the LCN2 sequence was inserted into the pDonor vector and transferred into the $\mathrm{pKO}$ vector using previously described methods ${ }^{21}$. The FLAG and SFB tags were added in front of the N-terminus of LCN2.

All of the constructs were confirmed by both DNA sequencing and diagnostic digestion.

\section{Cell culture and transfection}

The human oral squamous cell carcinoma cell lines CAL-27 and SCC-9 and the lentivirus vector packaging cell line HEK293t were obtained from the American Type Culture Collection (ATCC). The CAL-27RE cell line, a resistant strain of the CAL-27 cell line, was gifted from Professor Li (Sun Yat-sen Memorial Hospital, Sun Yat-sen University, China). All OSCC cell lines were routinely cultured in DMEM and DMEM-F12 medium supplemented with $10 \% \mathrm{FBS}$ in a $37-{ }^{\circ} \mathrm{C}$ humidified incubator containing $5 \% \mathrm{CO}_{2}$. All the cell lines were validated by short tandem repeat profiling analysis and were free of mycoplasma contamination.

The transient transfection of OSCC cells was performed using Lipofectamine 3000 (Invitrogen) reagent according to the manufacturer's instructions.

For transient transfections using small interfering RNAs (siRNAs), siRNAs targeting VDR, RPS3 and LCN2 were synthesised by GenePharma (Suzhou, China). The transfection was performed with Lipofectamine 3000 reagent (Invitrogen, Carlsbad, USA) according to the manufacturer's protocol. The siRNA sequences are shown as follows:

siRNA sequences for LCN2, VDR and RPS3

\begin{tabular}{llll}
\hline Gene & Forward & Reverse \\
\hline RPS3 & s1 & CCAGGACAGAAAUCAUUAUTT & AUAAUGAUUUCUGUCCUGGTT \\
& s2 & GGUUGUGGUGUCUGGGAATT & UUUCCCAGACACCACAACCTT \\
LCN2 & s1 & CCUCCGUCCUGUUUAGGATT & UUCCUAAACAGGACGGAGGTT \\
& s2 & GAGCUGACUUCGGAACUAATT & UUAGUUCCGAAGUCAGCUCTT \\
VDR & si & CCUGCUCAGAUCACUGUAUTT & AUACAGUGAUCUGAGCAGGTT
\end{tabular}

For stable expression, lentiviral plasmids harbouring the desired gene were first transfected into 293T cells together with the packaging plasmids pSPAX2 and pMD2.G at a ratio of 5:3:2. HEK293 cells were placed into a $10-\mathrm{cm}$ plate and cultured as previously described. After reaching $70-80 \%$ confluence, the cells were transfected with $6 \mu \mathrm{g}$ psPAX2, $3 \mu \mathrm{g}$ pMD2.G and $10 \mu \mathrm{g}$ transfer vector using Lipofectamine 3000 reagent. Fortyeight hours after transfection, the supernatants of each group were collected and used to infect OSCC cells for another $48 \mathrm{~h}$. Puromycin-tolerant OSCC cells were picked. Subsequent western blotting and PCR were applied to confirm the correct expression of the stable cell lines.

\section{OSCC sample collection and patient follow-up}

To address the research purpose, patients presenting at the Department of Oral and Maxillofacial Surgery, Sun Yat-sen Memorial Hospital between 2011 and 2013 for the treatment of OSCC were recruited. Inclusion criteria included a pathological diagnosis of OSCC and willingness to participate in the subsequent follow-up. Patients were excluded as study subjects if they were diagnosed with multiple cancer or other severe diseases. Data on the features of the OSCC patients, including age, gender, tumour differentiation, lymphatic metastasis and clinical stage, were collected. All patients would have a referral at least every season. In addition, their tumour samples and adjacent noncancerous (ANC) samples were collected. The ANC tissue refers to an area at least $2 \mathrm{~cm}$ from the tumour lesion, representing the resection 
border, being pathologically confirmed as noncancerous tissue.

\section{In vivo tumour xenograft and drug application}

To explore the effects of vitamin D on OSCC tumours in vivo, CAL-27 cells and their LCN2-stable cell lines were applied for tumour xenografts. OSCC cells $(2.0 \times$ $10^{6}$ ) were implanted into the right upper backs of BALB/c nude mice (female, 5 weeks old). The tumours were measured every 3-4 days to determine the tumour volume $\left(\mathrm{mm}^{3}\right)$ calculated as length $\times$ width $^{2} \times 0.5$. The tumours reached $\sim 5 \times 5 \mathrm{~mm}$ (length $\times$ width, $60 \mathrm{~mm}^{3}$ ) after 3 weeks.

When the tumour volume reached $\sim 60 \mathrm{~mm}^{3}, 30 \mu \mathrm{g} / \mathrm{kg}$ $1,25 \mathrm{D} 3$ or $5 \mathrm{mg} / \mathrm{kg}$ cisplatin was intraperitoneally injected according to the treatment group. Based on the preexperimental results in vitro, vitamin D was supplied first, and then chemotherapy was initiated. The vitamin D administration interval was 2 days, and the chemotherapy administration interval was 4 days. Tumour volume was measured before each chemotherapy event.

Tumour formation was observed, and tumour growth curves were constructed. The mice were killed after 5 injections, and the tumours were harvested and then frozen or paraffin embedded for immunohistochemical detection.

\section{Immunohistochemistry (IHC)}

Immunohistochemical staining was performed according to standard protocols. After deparaffinization, antigen retrieval was conducted using $10 \mathrm{mM}$ sodium citrate buffer $(\mathrm{pH}$ 8.0) in a pressure cooker at full power for $5 \mathrm{~min}$. Briefly, the tissue sections were blocked sequentially with $3 \% \mathrm{H}_{2} \mathrm{O}_{2}$ and normal serum and then incubated with primary antibodies at $4{ }^{\circ} \mathrm{C}$ overnight. The tissue sections were incubated with a biotinylated secondary antibody and conjugated with a streptavidin-HRP complex (ready-to-use SP kit; Zhongshan Co., Beijing, China). Finally, the sections were visualised with 3-3'diaminobenzidine and counterstained with haematoxylin and mounted. The samples were rinsed with phosphatebuffered saline (PBS) between each step.

\section{Evaluation of IHC staining}

IHC tissue staining was evaluated as previously described $^{44}$ by 2 pathologists, who assessed the number of positive cells and the intensity of staining. The positive results were judged by semi-quantitative points. The staining intensity scores were 0 (negative), 1 (weak), 2 (medium) and 3 (strong). The percentage of positive cells was scored as 0 (0\%), 1 (1-25\%), 2 $(26-50 \%)$ and $3(>50 \%)$. The staining intensity score and the proportional score were added to obtain the total score. A total score $\geq 3$ was considered to represent high expression. A total score $<3$ was considered to represent low expression.

\section{Western blot, immunoprecipitation and mass spectrometry}

For protein extraction, the cells were washed twice with cool PBS, harvested by scraping and then lysed in lysis buffer (Beyotime, China). Following centrifugation, the supernatant was collected, and the protein concentration was determined using the BCA Protein Assay Kit (Pierce ${ }^{\mathrm{TM}}$, USA).

For western blotting, cell lysates were electrophoretically separated on an SDS-PAGE gel using a standard protocol. The proteins were then transferred to polyvinylidene fluoride (PVDF) membranes (IPVH00010; Millipore, USA). The membranes were blocked with $5 \%$ non-fat milk in Tris-buffered saline containing $0.1 \%$ Tween-20 (TBST) for $1 \mathrm{~h}$ at room temperature. The blots were then incubated with the antibodies mentioned above at $4{ }^{\circ} \mathrm{C}$ overnight, washed in TBST and then probed with secondary antibody. Western blot analysis was performed using the grey value statistics of the blots.

For immunoprecipitation, the supernatants were first incubated with S-protein agarose beads (\#69704, Millipore, for SFB-LCN2) overnight at $4{ }^{\circ} \mathrm{C}$, and the precipitates were washed three times with NETN buffer. To detect endogenous interactions, the clarified supernatants were incubated with the antibodies mentioned above for $2 \mathrm{~h}$ and then with magnetic beads (Pierce ${ }^{\mathrm{TM}}$ Protein A/G Magnetic Beads, \# 88802, Thermo Fisher overnight. After being washed three times with NETN buffer, the samples were collected and analysed by western blot.

Proteins immunoprecipitated by LCN2 antibody were digested, and the peptides were analysed by mass spectrometry.

\section{RNA extraction, real-time quantitative RT-PCR and RNA sequencing}

Total RNA was extracted using TRIzol reagent (Takara, Japan) according to the manufacturer's instructions and then reverse transcribed into cDNA using the PrimeScript $^{\mathrm{TM}}$ RT Master Mix (Takara, Japan) on an ABI 9700 Real-Time PCR system (ABI, USA). The newly synthesised cDNA was then used as a template for the detection of the desired gene.

Specifically, $1 \mu \mathrm{l}$ of cDNA was mixed with TB Green ${ }^{\circledR}$ Premix Ex Taq ${ }^{\mathrm{TM}}$ II (Takara, Japan) in a $20-\mu \mathrm{l}$ reaction. All of the reactions were run in triplicate using the primers described above. The reaction conditions were as follows: $94{ }^{\circ} \mathrm{C}$ for $2 \mathrm{~min}, 94^{\circ} \mathrm{C}$ for $20 \mathrm{~s}, 58^{\circ} \mathrm{C}$ for $20 \mathrm{~s}$ and $72^{\circ} \mathrm{C}$ for $20 \mathrm{~s}$, for 40 cycles. The relative expression of mRNA was detected using the Roche LightCycler 480 II Real-time PCR machine (Roche, USA). The primer sequences were as follows: 
Primer sequences for PCR

\begin{tabular}{lll}
\hline Gene & Forward & Reverse \\
\hline LCN2 & CCCGCAAAAGATGTATGCCA & TCTTAATGTTGCCCAGCGTG \\
VDR & GTGGACATCGGCATGATGAAG & GGTCGTAGGTCTTATGGTGGG \\
GAPDH & GAGTCAACGGATTTGTTCGT & GACAAGCTTCCCGTTCTCAG \\
RPS3 & AGAGGAAGTTTGTCGCTGATG & GCACCTCAACTCCAGAGTAGC \\
\hline
\end{tabular}

The cells of the CAL-27 cell line were pretreated with $30 \mathrm{nM}$ vitamin $\mathrm{D}$ for 3 days and then with $5 \mu \mathrm{M}$ cisplatin and/or $30 \mathrm{nM}$ vitamin D for $36 \mathrm{~h}$. RNA was extracted using TRIzol (Thermo Fisher Scientific). After removing rRNA, RNAs were fragmented and reverse transcribed using random primers. cDNAs were ligated with adaptors, amplified via $\mathrm{PCR}$ and then sequenced with an Illumina sequence analyser. Filtered sequences were aligned with HISAT, and gene expression was analysed by DEGseq software. The differentially expressed genes were subjected to Gene Ontology (GO) and KEGG pathway analysis and were used to create heatmaps with the pheatmap package of $R$.

\section{DNA isolation, bisulfite treatment and methylation-specific PCR}

Genomic DNA was isolated from OSCC cell samples with a DNA isolation kit (\#3101050, Simgen, China). The bisulfite treatment of DNA was performed by using an EZ DNA Methylation-Gold ${ }^{\mathrm{TM}}$ Kit (\#D5055, ZYMO Research, USA) according to the manufacturer's instructions.

\section{Quantitative methylation-specific PCR}

Quantitative methylation-specific PCR (qMSP) was performed as described previously ${ }^{31}$. Briefly, genomic DNA was isolated with a Genomic DNA Purification Kit (Thermo Fisher Scientific, Cat. \#K182104A) according to the manufacturer's methods. For each sample, $0.8 \mu \mathrm{g}$ of genomic DNA was treated with sodium bisulfite using the EZ DNA Methylation-GOLD Kit (ZYMO Research, USA, Cat. \#D5008) following the manufacturer's instructions. The resulting genomic DNA was subjected to PCR analysis with the following primers:

LCN2-methylated primer sequences

LCN2

\begin{tabular}{lll} 
Methylated & Forward & CGAGAGTTATTGCGTTIAGTCGA \\
& Reverse & CGAATAAATCACGAAATCAAAAATTCGA \\
Unmethylated & Forward & AGAGTTATTGTGTTAATTGAGGA \\
& Reverse & CAAATAAATCACAAAATCAAAAATTCAA \\
\hline
\end{tabular}

Methylation-specific PCR (MSP) was performed according to the MSP Kit (\#R100A, Takara, Japan) protocol. Specificity, $2 \mu \mathrm{g}$ of genomic DNA was used for sodium bisulfite treatment, and two sets of primers specific to the methylated and unmethylated target sequences were used in two PCRs. PCR products (a 273-bp product, both methylated and unmethylated) were separated by $3 \%$ agarose gel electrophoresis.

\section{Flow cytometry}

For the flow cytometric quantification of cell death, the cells were treated as indicated. Then, cells were collected, washed twice with PBS and stained using Annexin VFITC/PI (\#556547, BD) according to the manufacturer's instructions. Stained cells were analysed using a Becton Dickinson FACScan Flow Cytometer (FACScan, BD), and data were processed using FlowJo software.

\section{Cytotoxicity assay}

Relevant cells were plated into 96-well plates (5000 cells/well) and cultured $24 \mathrm{~h}$ before treatment. Then, cell death was measured at the indicated time points with the LDH assay using a CytoTox 96 Non-Radioactive Cytotoxicity Assay Kit (\#G1781, Promega) according to the manufacturer's protocol.

The LDH release rates were determined following the protocol of the Cell-Mediated Cytotoxicity Assay kit:

\footnotetext{
$\%$ Cytotoxicity $=\frac{\text { Experimental }- \text { Effector spontaneous }- \text { Target spontaneous }}{\text { Target maximum }- \text { Target spontaneous }} \times 100$
}

\section{Cell proliferation assay}

At $24 \mathrm{~h}$ after transfection, the cells were collected, and 2000 cells/well were plated into 96-well plates. The number of cells at 24, 48 and $72 \mathrm{~h}$ was determined using the MTS Assay Kit (\#G3580, Promega, USA). The medium was removed from each well, and $100 \mu \mathrm{l}$ of $10 \%$ MTS in DMEM was added. The plates were incubated for an additional $2 \mathrm{~h}$, and absorbance at $490 \mathrm{~nm}$ was measured using a microplate reader (Multiskan MK3, China). The data were presented as original OD values.

\section{Cisplatin cytotoxicity assay}

At $24 \mathrm{~h}$ after transfection, OSCC cells were seeded at 10,000 cells/well into 96-well plates. After being incubated overnight, the cells were treated with one of various concentrations $(0,2.5,5,10,20,40$, and $80 \mu \mathrm{mol} / \mathrm{L})$ of cisplatin (\#1134357, Sigma, USA), and the MTS assay was applied to examine the cytotoxicity of cisplatin after $48 \mathrm{~h}$ of treatment.

The relative cell survival (\%) was determined by the formula $(\mathrm{OD} \times \chi \mu \mathrm{mol} / \mathrm{L} / \mathrm{OD} 0 \mu \mathrm{mol} / \mathrm{L}) \times 100$, where $\mathrm{X}$ represents cisplatin concentration.

The percentage of cell mortality (\%) was then calculated as 100 - relative cell survival. 


\section{Statistical analyses}

All statistical analyses were conducted using SPSS 19.0 statistical software. Spearman correlation analyses were used to examine the correlation between LCN2 expression and cisplatin chemotherapy in patients who experience recurrence. An unpaired $t$ test was used to compare LCN2 expression in patients with primary or recurrent OSCC tissues. Kruskal-Wallis analysis was used to examine the relationships between clinicopathological characteristics and protein expression. The survival curves were plotted using the Kaplan-Meier method and compared with the log-rank test. Fisher's exact test was used to examine the relationships between LCN2 expression and xenograft features. Student's $t$ test was used to compare the PCR results, cell apoptosis, tumour xenograft results, and cell functions (proliferation, migration, invasion, etc.) between the different groups. Unless otherwise noted, quantitative data are expressed as the mean and standard error of the mean (S.E.M.). Statistical significance was determined with a paired Student's $t$ test. ${ }^{*} P<0.05$; ${ }^{* *} P<0.01$; ${ }^{* * * *} P<0.001$, compared with the control.

\section{Data availability}

The datasets used for the current study are available from the corresponding author on reasonable request. All data generated or analysed during this study are included in this published article or its supplementary information files (Figs. 1s-5s)

\section{Acknowledgements}

This work was supported by grants from National Natural Science Foundation of China (\#81772892, \#31801075), Science and Technology Program of Guangdong (\#2016A030313348, \#2018A030310344), Fundamental Research Funds for the Central Universities (\#16ykjc17), The Key Laboratory of Malignant Tumor Gene Regulation and Target Therapy of Guangdong Higher Education Institutes, Sun-Yat-Sen University (Grant KLB09001), and Key Laboratory of Malignant Tumor Molecular Mechanism and Translational Medicine of Guangzhou Bureau of Science and Information Technology ([2013]163).

\section{Author details \\ 'Guangdong Provincial Key Laboratory of Malignant Tumor Epigenetics and Gene Regulation, Sun Yat-Sen Memorial Hospital, Sun Yat-Sen University, Guangzhou, China. ${ }^{2}$ Department of Oral and Maxillofacial Surgery, Sun Yat-sen Memorial Hospital, Sun Yat-sen University, Guangzhou, China. ${ }^{3}$ Medical Research Center, Sun Yat-Sen Memorial Hospital, Sun Yat-Sen University, Guangzhou, China. ${ }^{4}$ Department of Pathology, Sun Yat-sen Memorial Hospital, Sun Yat-sen University, Guangzhou, China. ${ }^{5}$ State Key Laboratory of Oncology in South China, Sun Yat-Sen University Cancer Center, Guangzhou, China}

\section{Conflict of interest}

The authors declare that they have no conflict of interest.

\section{Ethical approval}

This research was conducted in accordance with international guidelines and the ethical standards outlined in the Declaration of Helsinki. This study was approved by the Sun Yat-sen Memorial Hospital Institutional Review Board. All animal experiments were conducted following the Ministry of Health national guidelines for housing and care of laboratory animals and performed in accordance with institutional regulations after review and approval by the Institutional Animal Care and Use Committee at Sun Yat-sen University.

\section{Publisher's note}

Springer Nature remains neutral with regard to jurisdictional claims in published maps and institutional affiliations.

Supplementary Information accompanies this paper at (https://doi.org/ 10.1038/s41419-019-2177-x).

Received: 5 September 2019 Revised: 21 November 2019 Accepted: 25 November 2019

Published online: 09 December 2019

\section{References}

1. Chen, W. et al. Cancer statistics in China, 2015. CA Cancer J. Clin. 66, 115-132 (2016).

2. Siegel, R. L., Miller, K. D. \& Jemal, A. Cancer statistics, 2017. CA Cancer J. Clin. 67 , 7-30 (2017).

3. Cohen, E. E. et al. Phase III randomized trial of induction chemotherapy in patients with N2 or N3 locally advanced head and neck cancer. J. Clin. Oncol. 32, 2735-2743 (2014).

4. Zhong, L. P. et al. Randomized phase III trial of induction chemotherapy with docetaxel, cisplatin, and fluorouracil followed by surgery versus up-front surgery in locally advanced resectable oral squamous cell carcinoma. J. Clin. Oncol. 31, 744-751 (2013).

5. Adamska, A. et al. Molecular and cellular mechanisms of chemoresistance in pancreatic cancer. Adv. Biol. Regul. 68, 77-87 (2018).

6. Clemens, E. et al. A comparison of the Muenster, SIOP Boston, Brock, Chang and CTCAEv4.03 ototoxicity grading scales applied to 3,799 audiograms of childhood cancer patients treated with platinum-based chemotherapy. PLoS ONE 14, e0210646 (2019).

7. Song, Y., Liu, Y., Lin, M., Sheng, B. \& Zhu, X. Efficacy of neoadjuvant platinumbased chemotherapy during the second and third trimester of pregnancy in women with cervical cancer: an updated systematic review and meta-analysis. Drug Des. Dev. Ther. 13, 79-102 (2019).

8. Hanel, A. \& Carlberg, C. Vitamin D and evolution: pharmacologic implications Biochem. Pharmacol. pii: S0006-2952 (2019). 30279-5 PMID: 31377232.

9. Skrajnowska, D. \& Bobrowska-Korczak, B. Potential molecular mechanisms of the anti-cancer activity of vitamin D. Anticancer Res. 39, 3353-3363 (2019).

10. Bostick, R. M. Effects of supplemental vitamin D and calcium on normal colon tissue and circulating biomarkers of risk for colorectal neoplasms. J. Steroid Biochem. Mol. Biol. 148, 86-95 (2015).

11. Santos, J. M. \& Hussain, F. VD3 mitigates breast cancer aggressiveness by targeting V-H(+)-ATPase. J. Nutr. Biochem. 70, 185-193 (2019).

12. Gilzad-Kohan, H., Sani, S. \& Boroujerdi, M. Calcitriol reverses induced expression of efflux proteins and potentiates cytotoxic activity of gemcitabine in capan-2 pancreatic cancer cells. J. Pharm. Pharm. Sci. 20, 295-304 (2017).

13. Liu, Y., Wang, X., Sun, X., Lu, S. \& Liu, S. Vitamin intake and pancreatic cancer risk reduction: a meta-analysis of observational studies. Medicine 97, e0114 (2018).

14. Bochen, F. et al. Vitamin D deficiency in head and neck cancer patients prevalence, prognostic value and impact on immune function. Oncoimmunology 7, e1476817 (2018).

15. Urashima, M. et al. Effect of vitamin D supplementation on relapse-free survival among patients with digestive tract cancers: the AMATERASU randomized clinical trial. JAMA 321, 1361-1369 (2019).

16. Huang, Z. et al. 1,25-Dihydroxyvitamin D3 alleviates salivary adenoid cystic carcinoma progression by suppressing GPX1 expression through the NFkappaB pathway. Int. J. Oncol. 48, 1271-1279 (2016).

17. Miyamoto, $\mathrm{T}$. et al. Lipocalin 2 enhances migration and resistance against cisplatin in endometrial carcinoma cells. PLOS ONE 11, e0155220 (2016).

18. Lund, R. J. et al. DNA methylation and transcriptome changes associated with cisplatin resistance in ovarian cancer. Sci. Rep. 7, 1469 (2017).

19. Xia, Y., Shen, S. \& Verma, I. M. NF-kappaB, an active player in human cancers. Cancer Immunol. Res. 2, 823-830 (2014).

20. Gan, X. et al. High GPX1 expression promotes esophageal squamous cell carcinoma invasion, migration, proliferation and cisplatin-resistance but can be reduced by vitamin D. Int J. Clin. Exp. Med. 7, 2530-2540 (2014).

21. Hu, K. et al. High-performance gene expression and knockout tools using sleeping beauty transposon system. Mob. DNA 9, 33 (2018). 
22. Hodgson, A. et al. Metalloprotease NleC suppresses host NF-kappaB/inflammatory responses by cleaving p65 and interfering with the p65/RPS3 interaction. PLoS Pathog. 11, e1004705 (2015).

23. Wu, M., El, Q.S. \& Hardwidge, P.R. SseL deubiquitinates RPS3 to inhibit its nuclear translocation. Pathogens 7, E86 (2018).

24. Dong, J. et al. Ribosomal protein S3 gene silencing protects against cigarette smoke-induced acute lung injury. Mol. Ther. Nucleic Acids $\mathbf{1 2}$ 370-380 (2018).

25. Tayyem, R. F., Mahmoud, R. I., Shareef, M. H. \& Marei, L. S. Nutrient intake patterns and breast cancer risk among Jordanian women: a case-control study. Epidemiol. Health 41, e2019010 (2019).

26. Abrahamsson, $\mathrm{H}$. et al. High level of circulating vitamin D during neoadjuvant therapy may lower risk of metastatic progression in high-risk rectal cancer. BMC Cancer 19, 488 (2019).

27. Bao, Y. et al. Stromal expression of JNK1 and VDR is associated with the prognosis of esophageal squamous cell carcinoma. Clin. Transl. Oncol. 20, 1185-1195 (2018).

28. Liu, X. et al. miR-1204 targets VDR to promotes epithelial-mesenchymal transition and metastasis in breast cancer. Oncogene 37, 3426-3439 (2018).

29. Yu, M. et al. Apolipoprotein M could inhibit growth and metastasis of SMMC7721 cells via vitamin D receptor signaling. Cancer Manag. Res. 11, 3691-3701 (2019).

30. Tai, J. et al. Up-regulated lipocalin-2 in pediatric thyroid cancer correlated with poor clinical characteristics. Eur. Arch. Otorhinolaryngol. 275 2823-2828 (2018).

31. Miki, M. et al. CLEC3A, MMP7, and LCN2 as novel markers for predicting recurrence in resected $\mathrm{G} 1$ and $\mathrm{G} 2$ pancreatic neuroendocrine tumors. Cancer Med. 8, 3748-3760 (2019).

32. Flanagan, J. M. et al. Platinum-based chemotherapy induces methylation changes in blood DNA associated with overall survival in patients with ovarian cancer. Clin. Cancer Res. 23, 2213-2222 (2017).
33. O'Hagan, H. M., Mohammad, H. P. \& Baylin, S. B. Double strand breaks can initiate gene silencing and SIRT1-dependent onset of DNA methylation in an exogenous promoter CpG island. PLoS Genet. 4, e1000155 (2008).

34. Tang, $Y$. et al. 1,25-dihydroxyvitamin-D3 promotes neutrophil apoptosis in periodontitis with type 2 diabetes mellitus patients via the p38/MAPK pathway. Medicine 97, e13903 (2018).

35. Nguyen-Yamamoto, L., Tanaka, K. I., St-Arnaud, R. \& Goltzman, D. Vitamin Dregulated osteocytic sclerostin and BMP2 modulate uremic extraskeletal calcification. JCl Insight 4, 126467 (2019).

36. Montenegro, K.R., Cruzat, V., Carlessi, R. \& Newsholme, P. Mechanisms of vitamin D action in skeletal muscle. Nutr. Res. Rev. 32, 192-204, https://doi.org/ 10.1017/S0954422419000064 (2019). Epub 2019 Jun 17 PMID: 31203824.

37. Zhou, W., Yuan, G. \& Wang, Q. Vitamin D attenuates lipopolysaccharideinduced inflammatory response in endothelial cells through inhibition of PI3K Akt/NF-kappaB signaling pathway. Pharmazie 74, 412-417 (2019).

38. Giridharan, S. \& Srinivasan, M. Mechanisms of NF-kappaB p65 and strategies for therapeutic manipulation. J. Inflamm. Res. 11, 407-419 (2018).

39. Sun, S. C. The noncanonical NF-kappaB pathway. Immunol. Rev. 246, 125-140 (2012).

40. Christian, F., Smith, E.L. \& Carmody, RJ. The regulation of NF-kappaB subunits by phosphorylation. Cells 5, E12 (2016).

41. de Castro, B. M. et al. NF-kappaB signaling pathway inhibitors as anticancer drug candidates. Anticancer Agents Med. Chem. 17, 483-490 (2017).

42. Tang, W. et al. Lipocalin 2 suppresses ocular inflammation by inhibiting the activation of NF-kappabeta pathway in endotoxin-induced uveitis. Cell Physiol. Biochem. 46, 375-388 (2018).

43. Stanborough, T., Niederhauser, J., Koch, B., Bergler, H. \& Pertschy, B. Ribosomal protein S3 interacts with the NF-kappaB inhibitor IkappaBalpha. FEBS Lett. 588 659-664 (2014).

44. Li, H. F. et al. Downregulation of MACC1 inhibits invasion, migration and proliferation, attenuates cisplatin resistance and induces apoptosis in tongue squamous cell carcinoma. Oncol. Rep. 33, 651-660 (2015). 